\title{
Review and investigations on future research directions of mobile based telecare system for cardiac surveillance
}

\author{
S. Palanivel Rajan \\ Assistant Professor \& Research and Development Cell Coordinator, Department of Electronics \& Communication Engineering, M. Kumarasamy College \\ of Engineering (Autonomous), Karur, Tamilnadu, India
}

Received 3 October 2014; accepted 8 May 2015

\begin{abstract}
Tele-surveillance is the method of patient monitoring, provided that medical practitioner and patient are separated by a large distance and it also involves inaccessibly monitoring the patients who are far away from the health care provider. This article talks about a few of the vital challenges and opportunities of communications research in the areas of Telemedicine and E-Health. Especially, we focus on some of the key communications challenges for understanding interoperable and future-proof of Mobile Tele-health systems, need for doing research in this broad area with the help of statistics collected from various health organizations and how drawbacks of some existing technologies can be solved. We have overviewed this telemetry technology and present the state of the art on each aspect. Some research problems that need to be solved in this area are also described. All Rights Reserved ( 2015 Universidad Nacional Autónoma de México, Centro de Ciencias Aplicadas y Desarrollo Tecnológico. This is an open access item distributed under the Creative Commons CC License BY-NC-ND 4.0.
\end{abstract}

Keywords: Biomedical communication; Biomedical signal processing; Biomedical telemetry; Electrocardiography; Healthcare and Telemedicine

\section{Introduction}

Tele-health is a fast-growing inter-disciplinary area, in which electronic information and communication technology is used to deliver health and medical information and services over large and small distances (Suman \& Prathyusha, 2012). Tele-health includes telemedicine, which offers empowerment, a better quality of life, and reduced cost of care for patients with chronic disease, such as cardiovascular disease, diabetes, chronic respiratory diseases, and cancer (Donoghue \& Herbert, 2006). However, Tele-health also includes applications for healthy people, who want to maintain or improve their health. Mobile Tele-health systems are becoming more important all the time, especially in the care of patients that are isolated or traveling, far from a reference hospital. These systems must be embedded in low cost, small devices with low power consumption, and should have an interface that is usable by the patient. Incorporating technologies such as Bluetooth and Global System for Mobile (GSM) allows the wireless transmission to health centers (Kumekawa, 2000).

The application of wireless communications is already quite common in hospital and emergency settings (Gao et al., 2008). Some examples of medical equipments include heart, blood

E-mail address: palanivelrajanme@gmail.com pressure and respiration monitors (Kumekawa, 2000). In addition, emergency medical service companies are, or will be, important users of telemetry and other wireless technology (Gao et al., 2008). In vehicles, telemedicine equipment can be as simple as a cell phone or a laptop computer with two-way videoconferencing capabilities. More sophisticated vehicles offer mounted video cameras that the hospital emergency physician can pan and zoom remotely (Gao et al., 2008).

Another very common form of telemedicine that has generated a lot of interest in the networking community is remote patient monitoring (Rajan \& Sukanesh, 2013a), which facilitates an improved quality of life for the patient by enabling the measurements to be made anywhere there is a phone connection, and by reducing the need for routine trips to the clinic. While such systems have demonstrated positive outcomes, e.g. in terms of reduced number of trips to the emergency room (Kumekawa, 2000), wireless monitoring promises further improvements by providing continuous monitoring, patient mobility, and improving patient compliance with frequent and better quality measurements (Kumekawa, 2000). When appropriate health professionals cannot be physically present to diagnose patients, telecommunications technology can be used to connect these professionals to those in need of their expertise (Qureshi et al., 2005). In hospitals and homes, telemedicine has been shown to reduce the cost of health-care and increase efficiency through better management of chronic diseases, shared 
health professional staffing, reduced travel times, and fewer or shorter hospital stays (Xiao et al., 2000).

We focus on using telemedicine to improve outcomes when dealing with acute incidents involving critical care patients. By relaying real-time telemetry and video from ambulances, we hope to provide expert opinions on complex injuries, and to aid the inhospitalities in better preparing themselves for incoming patients. In many situations, the timely application of an appropriate therapy is of critical importance, and mobile telemedicine can help (Xiao et al., 2000). Patients with heart arrhythmia usually need to be monitored and controlled in hospital for one to several days. These patients are treated to reach on normalizing their heart arrhythmia or achieve an average heart frequency (Qureshi et al., 2005). Sometimes it is necessary to monitor some heart patients in longer periods of time to provide more certain documentation for the treatment's correctness, but the patients often are released from the hospital to give the priority to other heart patients on the waiting list, whom need to be hospitalized immediately. Furthermore, some of heart medicaments are effective only when the patients have minimum activity, namely, when they are hospitalized, but these medicaments are not so effective at home, where the patients have normal or higher activity level. If the ECG or heart rate crosses the threshold set by the doctor depending on the patient, a Short Messaging Service (SMS) is generated by Microcontroller unit automatically and it will be transmitted to the doctor's mobile through the GSM modem. The SMS gives the ECG and heart rate of the patient so that medical aid can be rushed to the spot.

This paper describes the issues in developing a low cost, portable system with wireless transmission capabilities for the acquisition, processing, storing and visualization in real time of the heart rate to a mobile phone and how the problems can be solved.

\section{Background}

National Health and Nutrition Examination (NHANES) that surveys that the prevalence of low coronary heart disease risk, overall and by sex in the year of 1971-2006 stated that $80 \%$ of people are affected. Survey of 2009-2010 has showed the statistics of diabetes mellitus awareness, treatment and control. Also diabetes can lead to cardiovascular disease, blindness and kidney failure. The treated and controlled occupied a percent- age of $25.8 \%$. Then the treated and uncontrolled occupied a percentage of $43.2 \%$ and not treated but aware was $8.3 \%$ and not treated and undiagnosed had 22.7\% (NHANES, 20092010). World Health Organization (WHO) annual statistics report includes information from 194 countries on the percentage of men and women with raised blood pressure and blood glucose levels. Another important issue is obesity. In regions of the world, obesity doubled between 1980 and 2008 (as per the statement from Dr. Ties Boerma, Director, Department of Health Statistics and Information Systems, WHO). Today, half a billion people (12\% of the world's population) are considered obese. Highest obesity levels are in the WHO Region of the Americas (26\% of adults) and the lowest in the WHO SouthEast Asia Region (3\% obese).

Heart attack (also called myocardial infarction) is the death of the heart muscle due to loss of blood supply caused due to blockage in one or more of the coronary arteries supplying the heart. Heart disease is the leading cause of death in the United States. More than a million persons in the United States have heart attack each year and about $50 \%$ of them had passed away. The risk of heart attack in both men and women are almost equal, with women accounting for nearly half of all heart attack deaths (Table 1). According to a projection by the World Health Organization (WHO) and the Indian Council of Medical Research (ICMR), India will not only be the heart attack capital but also the capital of diabetes and hypertension by 2020 . People having heart disease had increased from $1-2 \%$ to $3-5 \%$ in rural India and from $2-3 \%$ to $10-11 \%$ in urban India. This represented an overall increase of 300 percent over the past 30 years. In 2006 (baseline data for the 2020 Impact Goals on Cardiovascular Diseases), age-standardized death rate from all cardio-vascular diseases (CVDs) was 262.5 per 100,000. Death rates from heart diseases and other cardiovascular causes were 43.6, 200.2, and 53.9 per 100,000, respectively. Data from NHANES 1999-2010 reveal that, overall, 8.1\% of Americans self-reported having some type of CVD. Among those with CVD, risk factor prevalence, awareness, treatment, and control were variable. Nearly $37 \%$ were current smokers or had quit for less than 12 months. From 2005 to 2010, the Data from the NHANES indicate that $33.5 \%$ of US adults $\geq 20$ years of age have hypertension. The prevalence of hypertension is nearly equal between men and women. African American adults have among the highest rates of hypertension in the world at $44 \%$.

Table 1

General Statistics (from WHO, ICMR) of Heart Attacks for Different Age People.

\begin{tabular}{|c|c|c|c|}
\hline Age & Criteria & Statistics of Men & Statistics of Women \\
\hline Does not matter & Vulnerability to heart attacks & $\begin{array}{l}\text { Men are more vulnerable to heart attacks as } \\
\text { compared to women } \\
\text { More than } 420,000 \text { men are hospitalized for heart } \\
\text { attacks every year }\end{array}$ & $\begin{array}{l}\text { Though women are less vulnerable to heart attacks, } \\
\text { deaths due to heart attacks are more in women as } \\
\text { compared to men. Around } 350,000 \text { women are } \\
\text { hospitalized for heart attacks every year }\end{array}$ \\
\hline 40 and above & Death after first heart attack & $\begin{array}{l}\text { In this age group, around } 18 \% \text { deaths occur after } \\
\text { the first heart attack }\end{array}$ & $\begin{array}{l}\text { In this age group, around } 22 \% \text { of women deaths } \\
\text { occur after the first heart attack }\end{array}$ \\
\hline 45 and above & Death after first heart attack & $\begin{array}{l}\text { In this age group, } 32 \% \text { deaths occur after first heart } \\
\text { attack }\end{array}$ & $\begin{array}{l}\text { For women in this age group, the fatality rate is } \\
\text { around } 42 \%\end{array}$ \\
\hline 45 and above & Risk of heart attack & $\begin{array}{l}\text { In men, risk of heart attack increases greatly after } \\
45 \text { years of age }\end{array}$ & $\begin{array}{l}\text { In women, risk of heart attack increases greatly } \\
\text { after } 50 \text { years of age }\end{array}$ \\
\hline 64 to 70 & Average age for first heart attack & Average age for first heart attack in men is 64 & Average age for first heart attack in women is 70 \\
\hline
\end{tabular}


Among hypertensive adults, $80 \%$ are aware of their condition, $71 \%$ are using antihypertensive medication, and only $48 \%$ of those aware that they have hypertension have their condition controlled. For four decades of progress, in 2010 the Americans are greater than 18 years of age, $23.1 \%$ of men and $18.3 \%$ of women continued to be cigarette smokers. In 2009, $19.5 \%$ of students use tobacco.

The rate of death increases every year due to the modern life style. The myocardial infarction happens due to large stress, strain, increased fat, increased chemical content in the body, blockages in the blood vessels, obesity, etc. Awareness and treatment of hypertension were approximately $80 \%$, but only two-thirds of those who were treated were controlled to goal levels. Awareness and treatment of hypercholesterolemia were $60 \%$ and $68 \%$, respectively, and $80 \%$ of treated individuals were at goal cholesterol levels. More than three fourths were overweight or obese, and $45 \%$ were obese; $18 \%$ had diabetes mellitus; $45 \%$ participated in no physical activity; $100 \%$ of those with CVD met three or fewer of the five components of the healthy diet score. The following age-adjusted prevalence estimates from the National Health Interview Survey (NHIS), National Center for Health Statistics (NCHS), are for diagnosed conditions for people $\geq 18$ years of age in 2011 .

Statistics related heart attacks, by age, reveal that this ailment leads to more deaths as compared to various other serious ailments. Family history, work related stress, lack of proper sleep, smoking, drinking excessively and lack of exercise are the main reasons for heart attacks. As per the 2012 update provides critical data about cardiovascular quality of care, procedure utilization, and costs. In light of the current national focus on healthcare utilization, costs, and quality, it is critical to monitor and understand the magnitude of healthcare delivery and costs, as well as the quality of healthcare delivery, related to CVDs. By 2020 the WHO estimates nearly 25 million CVD deaths, and heart disease will be the leading cause of death throughout the world. Between 1990 and 2020, deaths from non-communicable diseases and injury are expected to rise from 33 million to 58 million annually, with a similar proportional increase in years of life lost. CVD causes 8.5 million deaths among women annually. It is the largest single cause of mortality among women, accounting for one third of all deaths in women worldwide.

In developing countries, half of all deaths of women over 50 are due to heart disease. In the year 2012, more than 920,000 people will have a heart attack nearly half of them will occur without prior symptoms or warning signs. 250,000 people die annually of sudden cardiac death (i.e. 680 every day of the year). One half of the victims of sudden cardiac death are under the age of 65 . An estimated 80 million people have one or more types of heart disease. About 8.9 million Indians have chest pain (angina) caused by reduced blood flow to the heart muscle which occurs when the coronary arteries become blocked with a build-up of plaque. Currently about 7.9 million Indians are alive who have had a heart attack. Heart disease is a real killer, about 500,000 people die annually due to heart disease. In fact, it is the leading cause of death for both men and women. Among the types of heart disease, coronary heart disease is the most common type, with about $75 \%$ of heart disease elated deaths stemming from this type.

The incident rate of heart attacks per 10,000 people is around 190 in people who are over 85 years of age, around 117 for those between 75-84 years of age, 66 for those between 65 and 74 years of age, 37 for those between 55 and 64 and 18 for people between 45 and 54 . India have revealed that heart ailments take most lives in both urban and rural areas.

Heart rate frequency is very important health status information. Approximately 50,000 new cases are diagnosed each year. Heart failure is responsible for 11 million physician visits each year and more hospitalizations than all forms of cancer combined. Heart failure contributes to approximately 287,000 deaths a year. Also the statistical update of 2012 is a major source for monitoring both cardiovascular health and disease in the population.

In India, heart disease is the single largest cause of death in the country with heart attacks being responsible for one third of all deaths caused by heart diseases. According to a projection by the WHO and ICMR, one fifth of the deaths in India are from coronary heart disease. By the year 2020, it will account for one third of all deaths. There are an estimated 45 million patients of coronary artery disease in India. Physical inactivity increases all causes of mortality, and doubles the risk of CVD. Survival to hospital discharge among children with non-traumatic cardiac arrest is $8.6 \%$ and of bystander-witnessed ventricular fibrillation is $62.5 \%$. Most sudden deaths in athletes were attributable to CVD (56\%). Of the cardiovascular deaths that occurred, $29 \%$ occurred in blacks, $54 \%$ in high school students, and $82 \%$ with physical exertion during competition training, and only $11 \%$ occurred in females, although this proportion has increased over time.

A longitudinal study of 17 to 24 years of age students participating in National Collegiate Athletic Association sports showed that the incidence of non traumatic out-of-hospital cardiac arrest was 1 per 22,903 athlete participant years. Data from the Registrar General of India reported greater age-adjusted cardiovascular mortality in southern and eastern states of the country. Coronary heart disease (CHD) mortality is greater in south India, while stroke is more common in the eastern Indian states. CHD prevalence is higher in urban Indian populations, while stroke mortality is similar in urban and rural regions. Case-control studies in India have identified that the common major risk factors account for more than $90 \%$ of incident myocardial infarctions. The average annual rates of first cardiovascular events rise from 3 per 1000 men at 35 to 44 years of age to 74 per 1000 men at 85 to 94 years of age. For women, comparable rates occur 10 years later in life.

Male is more affected than female. Children were affected by birth as a congenital anomaly. In 2012 overall rate of death attributable to cardiovascular disease (International Classification of Diseases, 10th Revision, codes $\mathrm{I}_{00}-\mathrm{I}_{99}$ ) was 244.8 per 100,000. From 2002 to 2012, the rate of death attributable to CVD declined 30.6\%. Mortality data for 2012 show that CVD $\left(\mathrm{I}_{00}-\mathrm{I}_{99} ; \mathrm{Q}_{20}-\mathrm{Q}_{28}\right)$ accounted for $32.8 \%(811,940)$ of all 2,471,984 deaths in 2012. The percentage of the non smoking population with detectable serum cotinine declined from 52.5\% in 1999 to 
2012 to $40.1 \%$ in 2007 to 2008 , with declines occurring, and was higher for those 3 to 11 years of age (53.6\%) and those 12 to 19 years of age $(46.5 \%)$ than for those 20 years of age and older $(36.7 \%)$.

\section{Discussion on Existing System}

\subsection{Summary of Existing Methodologies}

After a robust literature survey related to this research work, it is found that all the existing patient monitoring systems are Internet or Web based. Generally by comparing with the old patient monitoring systems, a centralized server was required for gather bio-signals from the patient (Toral et al., 2001). For a test case the information acquired from the bio-signals are shared between the doctor and patient within a short range since it is based upon establishing a communication link through Bluetooth (Rasid \& Woodward, 2005). As per the details in Encyclopedia of surgery, the Electronic mail applications, doctors can able to know the abnormality detection of the post operative patients. In that above mentioned work (Elena et al., 2002), continuous monitoring of cardiac patients was not possible.

A thorough review of existing approaches toward developing wireless biological sensors has been covered in Woodward et al. (2001). These approaches can be divided into two major categories: fully analog and microcontroller-based. Fully analog systems are either: $a$ ) fully integrated amplifiers and transceivers, or b) assembled multi-chip modules. Although the fully integrated devices demonstrated in Lupu (2003), Widya et al. (2003), and Dong and Zhu (2004), and feature very small size and low power consumption (approximately 2-14 mW), they do not provide digital-signal filtering or bidirectional communications, except for the system described in Dong and Zhu (2004), which can modulate the inductive power link as a carrier signal for communication from the base station to the sensor. In addition, in order to compare the performance and capabilities of the fully analog systems, it would be useful to define a figure of merit as the analog telemetry efficiency factor (ATEF) (Lupu, 2003).

The existing systems create an ECG sensor system that can be wirelessly connected to a handheld device that can graphically present the heart signals (Ogawa et al., 2010).

During the second phase, analyzing the heart rate of the patients is achieved by fixing a threshold using Laboratory Virtual Instrumentation Engineering Workbench (LabVIEW) as a tool and to trigger an alarm during panic conditions to the nurses in hospitals (Rajan, 2012). Another existing model makes use of non-contacting copper foil electrodes (Hu et al., 2009). The electrodes are wrapped around the infusion supply polyvinyl chloride (PVC) tube from the solution bag, the drip chamber, and the infusion PVC tube from the drip chamber (Voskarides et al., 2003). Drip infusion fluids have electrical conductivity, so a capacitor is formed between the infusion fluid and each electrode. When an infusion fluid drop is forming, its length and diameter, and therefore the drip chamber capacitance, are increasing, causing change in the output signal (Dong \& Zhu, 2004). But these existing models do not provide accurate results as produced by the infrared sensors and these do not offer any means to alert the nurses directly during the night hours, as the alarm is placed near the patient. It requires additional labor to inform nurses about the panic condition of the patients. The other proposed works (Tahat, 2008) makes use of the concept of inducing an AC voltage in the patient's body by a pulse oscillator which is then recorded by the electrodes in the drip tube (Rajan \& Rajamony, 2013b). If the injection needle is detached the AC voltage changes and an alert is given to the nurse station. Even though some of the tele-health system are mobile based, it requires a centralized monitoring server (Widya et al., 2003). It should be noted that the system becomes unavailable, if the server fails.

\subsection{ECG abnormalities survey and discussion}

This survey part is purely based on the article (Kappiarukudil \& Ramesh, 2010). Cardiac abnormalities were common in the stress filled life style. Actually 9221 ECGs were analyzed, only 112 (less than 1.5\%) were normal, 1483 (16.08\%) showed only minor abnormalities and 7626 (82.7\%) showed major abnormalities. Atrial fibrillation (AF) \pm atrial flutter was present in 2336 patients (25.33\%). Excluding patients with paced rhythm (494 patients), QRS prolongation $\geq 120 \mathrm{~ms}$ was observed in 2378 patients (26\% of non-paced patients). Only 1569 (17.02\%) ECGs had pathological Q-waves indicative of myocardial infarction but may have been obscured by bundle branch block or paced rhythm. Of patients with T-wave abnormalities, all but 46 had another associated ECG abnormality. Of patients with moderate to severe T-wave changes, 1698 (20.25\%) patients were treated with digoxin, which was rather less than in the overall population. Of the 5718 patients for whom an assessment of cardiac function was available, only 67 (1.17\%) ECGs were normal, 697 (12.19\%) had minor and $4954(86.64 \%)$ had major abnormalities $\mathrm{AF} \pm$ atrial flutter was present in 1378 patients $(24.1 \%)$ and was more common in those with major valve abnormality but no major left ventricular systolic dysfunction (MLVSD). Excluding those with paced rhythm, a broad QRS complex ( $\geq 120 \mathrm{~ms})$ was observed in $1479(25.86 \%)$ patients and was more common in those with MLVSD compared to those with a major valve abnormality but no MLVSD, mild echocardiographic abnormality or no echocardiographic abnormality.

ECG appears to have limited utility in refuting the presence of high fidelity (HF) in a secondary care setting. Very few patients had a normal ECG and therefore even though a completely normal ECG made a diagnosis of MLVSD very unlikely, in agreement with previous reports, it was of little practical assistance (Kappiarukudil \& Ramesh, 2010). Moreover, 20\% of patients with a normal ECG had some form of major structural heart disease (MSHD). Although T-wave abnormalities were one of the most common abnormalities, they rarely occurred in isolation and did not seem more prevalent amongst patients on digoxin. Excluding T-wave changes from the classification of the ECG would have made little difference to our findings. MSHD was common, as might be expected in this study population. However, no single ECG variable increased the probability of MSHD to $>90 \%$ (a specific test) or reduced it to $<20 \%$ (a sensitive test). Major ECG abnormalities, particularly broad 
QRS with a left bundle branch block (LBBB) pattern and anterior Q-waves, increased the probability of finding MLVSD to $>70 \%$ and multivariable models improved diagnostic accuracy further but still lacked precision. These results suggest that the ECG, used alone, is of limited practical clinical use as a screening tool for selection of patients with suspected heart failure for cardiac imaging in a secondary care setting (Kappiarukudil \& Ramesh, 2010).

The prevalence of MSHD in patients with suspected heart failure in primary care is much lower than in our population and therefore the clinical utility of the ECG in that setting may be very different. Previous studies (Part-III) investigating the role of the ECG in primary care were not large, provided insufficient detail on ECG classification and focused solely on MLVSD. However, a recent meta-analysis of studies in primary care concluded that the ECG was an inadequate screening tool because of variable sensitivity, possibly due to variations in ECG classification, and low specificity. Screening for MSHD combining data from natriuretic peptides and the ECG may improve diagnostic accuracy. The ECG is complex to interpret and requires years of training for expert evaluation. Simplifying interpretation by focusing on easily quantified components that carry important diagnostic information might enhance the diagnostic utility of the ECG. QRS duration is easy to identify, objective, quantitative and the ECG variable most strongly related to left ventricular ejection fraction (LVEF), especially if the conduction pattern is taken into account. Therefore, focusing on QRS duration rather than whole ECG may simplify analysis and lead to less operator-dependent results (Kappiarukudil \& Ramesh, 2010).

The 12-lead ECG appears also to have limited value for the identification of ischaemic heart disease as the cause of heart failure. Ischaemic heart disease was present in $64 \%$ of patients on the basis of angiography or a history of myocardial infarction but only $24 \%$ of patients with MLVSD had pathological Q-waves. In $36 \%$ of patients with possible myocardial infarction the diagnosis was confounded by the presence of conduction abnormalities. Other patients will have left ventricular systolic dysfunction (LVSD) due to non-transmural infarction or myocardial hibernation. Real clinical utility of the ECG in heart failure appears not to be for diagnosing the presence or aetiology of MSHD, but rather for the identification of important complications that influence the choice of treatment. AF was present in $27 \%$ of patients. These patients should receive anti-coagulants, unless contraindicated, and require adequate ventricular rate control. QRS duration was $\geq 120 \mathrm{~ms}$ in $37 \%$ of patients with MLVSD, a rate similar to that observed in large clinical trials indicating the need for evaluation of device therapy, including implantable defibrillators and cardiac resynchronization therapy. Also, the ECG provides an accurate, objective and verifiable measure of heart rate that may be used to guide both pharmacological and device therapy.

Our analysis has a number of limitations. The method of data collection was designed to reduce patient-selection bias, but may not always have been effective. However, the survey enrolled a large number of patients over a short time and their characteristics were close to epidemiological predictions. This study was designed to capture information after the hospital episode was complete to prevent interference with usual clinical practice. Consequently, many patients with suspected HF did not undergo cardiac imaging as recommended in guidelines, particularly those without gross ECG abnormalities, and it seems reasonable to suppose that they would be less likely to have MSHD and MLVSD. Had they undergone imaging, it might have improved the diagnostic performance of the ECG (Kappiarukudil \& Ramesh, 2010). A prospective study design might have produced a different result, but would have brought its own biases. One other observation deserves mention. The Euro Heart Failure survey is an agreement with many other hospital discharge surveys that suggest that only about half of patients have LVSD. There is a widespread perception that the other half has diastolic heart failure. However, the clinical diagnosis of heart failure with a normal ejection fraction should not be equated with diastolic heart failure. Valve disease is another major cause of heart failure as are poorly controlled hypertension and atrial fibrillation. Many of these patients probably have abnormal long-axis systolic function. The classification of major structural heart disease adopted in this survey is, we believe, a useful template for use in future surveys. In this classification, diastolic LV dysfunction severe enough to cause heart failure appears rather infrequent. Ultimately, natriuretic peptides may be a better guide to diagnosis and outcome for this group of patients than echocardiography (Kappiarukudil \& Ramesh, 2010).

In conclusion, the 12-lead ECG is an important tool for the evaluation of patients with HF after the diagnosis is made. Its value as a screening or diagnostic tool in patients at low, medium or high risk of heart failure has yet to be proved (Kappiarukudil \& Ramesh, 2010).

\section{Methodology of This Research}

From the above discussion, it is very clear that the human death due to various CVD has been drastically increased due to changes in life style, especially in food habits. Hence it is essential to monitor the cardiac status continuously. Cardiac monitoring allows for 24-hour visualization of the patient's electrical activity within the heart, but typically limits the views of the electrical activity to two views at any one time. When monitoring a patient's electrical impulse flow through the heart with cardiac monitoring or a 12-lead ECG, proper placement of the electrodes becomes essential to obtain accurate data. Patients with heart arrhythmia usually need to be monitored and controlled in hospital for one to several days. Sometimes it is necessary to monitor some heart patients for longer periods of time to provide more certain documentation for the treatment's correctness, but the patients often are released from the hospital to give the priority to other heart patients on the waiting list whom need to be hospitalized immediately. The post-operative patients can develop complications once they are discharged from the hospital. In some patients the cardiac problems may re-occur when they start doing their routine work. Hence the ECG of such patients needs to be monitored for some time after their treatment. 
This helps in diagnosing the improper functioning of the heart and take precautions. Mobile telemedicine systems are becoming more important all the time, especially in the care of patients that are isolated or traveling, far from a reference hospital. The amplitude, duration, and rhythm of the QRS complex are used to aid cardiologists to identify abnormal functions of the heart. This survey stimulates the research work towards the design and development a model that detects the abnormal interpretations in the ECG and heart rate of a patient in advance thereby reducing the critical level of the patient by following precautionary measures at an earlier instant.

\section{Highlights of This Research Survey}

After a keen literature survey, it is clearly understood that what are the deviations should be included and enhancement in the existing methodologies/systems and the highlights of future work must include those showed in Table 2.

- Does not require any Internet facility.

- No need for any centralized server.

- To sense the painless heart attacks, especially for diabetic patients.

- Provides an alert about abnormal ECG/heart rate of the patient to the doctor in well advance.

- Monitoring of the patients by the doctor is achieved since the doctor's mobility is enhanced.

- To reduce the grave risk level of patient.

- Low fault tolerance level is $\leq 10 \%$.

- Portable and easy to handle.

- Less time consumption.

- Low power consumption model.

- Cost-effective model.

- Well suited for remote areas.

\section{Research Contributions in This Article}

To understand the motivation, background and need for the development of mobile based tele-health system. Statistics collected from WHO, ICMR, NHANES, NCHS, NHIS, etc., are included in this paper which helps to understand the social relevance of this system. A robust literature survey was made and it was very much helpful for analyzing the drawbacks/demerits in the existing systems (problem formulation). It enables the reader to set the goals of further research work in this broad area and have shown the direction to proceed it in further as tabulated in Table 2.

\section{Conclusions}

The increasing request of patients, suffering of chronic diseases, who wish to stay at home rather then in a hospital and also the increasing need of homecare monitoring for elderly people, have lead to a high demand of wearable medical devices. Also, extended patient monitoring during normal activity has become a very important target. Low power consumption is essential in continuously monitoring of vital signs. A survey on wireless cardiac tele-health system has been presented for future research directions. The most important value of this mobile tele-health system lies in the detection of ECG and heart rate of patients who are located in the remote areas or in travel and are not in a position to report to the doctor for immediate treatment, an alert SMS can be transmitted using the GSM technology to the doctors and advises can be sought for saving the life of the patient. This system could be used as a forewarning system for monitoring during normal activity or physical exercises. It enables the cardiac patient to be on the move globally carrying out his day to day work. It is also clear that the system can be used out of hospital settings. In clinical application also, we found that it can detect the difference between

Table 2

Deviations of Mobile Tele-Health System From Existing Monitoring System.

\begin{tabular}{|c|c|c|}
\hline System No & Existing System & Possible Future Enhancements \\
\hline 1 & $\begin{array}{l}\text { Web based system (i.e) Internet based. For example, to send the } \\
\text { information websites like, www.way } 2 \text { sms.com is used here }\end{array}$ & $\begin{array}{l}\text { No need for such internet facility (Web). Only mobile devices and } \\
\text { communication should be used in this system }\end{array}$ \\
\hline 2 & Centralized server is required & Centralized server will not be required \\
\hline 4 & Common to all aged peoples (increased false alarm rate) & $\begin{array}{l}\text { But age tuner is present for changing the threshold limit, since heart } \\
\text { rate is not a constant value }\end{array}$ \\
\hline 6 & For ECG pickup, 12 lead system is used & Sensors/electrodes with three lead configurations are used \\
\hline 7 & Bluetooth technology is used & GSM technology is used - coverage is more ( $2 \mathrm{G}$ or $3 \mathrm{G})$ \\
\hline 8 & Expensive (approx. $\$ 1000=$ Rs. 52,000 ) & Cost is very low $(<$ Rs. 52,000$)$ \\
\hline 9 & Applicable to only literate peoples & It is applicable to all levels of peoples (both literate and illiterate) \\
\hline
\end{tabular}


cardiovascular patients and normal persons. Its fault tolerance limit should be less than $\pm 10 \%$. It is inferred that the cost for designing this model is very low when compared to the existing patient monitoring system and the cost is approximately less than Rs. 46,000. Demonstration has been done for analyzing the processing time requirement for the system using different randomly selected ECG files on three different mobile phones using ten randomly selected ECG entries from MIT-BIH Arrhythmia database. This system enables the doctor to receive/ analyze the patient report and also deliver doctor's treatment and specialist advice to remote patient. The doctor doesn't need to be sitting in front of a stationary computer within the medical facility. Research needs to be done for finding more efficient methods for signal enhancement. By comparing this cardiac tele-monitoring system with the existing systems (as discussed in Part III), it is necessary to include lot of enhanced features which is required for the current scenario. These study results have great significance in researching, preventing and finding epidemics in cardiovascular system for the country.

\section{Future Scope}

Since most part of this paper is done as an offline analysis and efforts will be made it as an online process. In the future, this work will be served as a base that to use the GPS technology to exactly predict the location of the victim. The ECG sensor will activate the alarm sound and send the warning message through the Wi-Fi supported cellular phone (3G mobile phones). Better biosensors that reduces the noise when the person is in motion. This system also provides a way to analysis the ECG for a long-term. The principle of the development of science is that "nothing is impossible". So we shall look forward to a bright and sophisticated world.

\section{Acknowledgments}

Author of this paper would like to express his sincere thanks to the Department of Electronics and Communication Engineering, M. Kumarasamy College of Engineering (Autonomous), Karur, Tamilnadu, India, since it provided all the necessary facilities for the early development of wireless cardiac tele-health system.

\section{References}

Dong, J., \& Zhu, H.H. (2004). Mobile ECG detector through GPRS/Internet. In 17th IEEE Symposium on Computer-Based Medical Systems, 2004. CBMS 2004. Proceedings (pp. 485-489). IEEE.

Elena, M., Quero, J.M., Tarrida, C.L., \& Franquelo, L.G. (2002). Design of a mobile telecardiology system using GPRS/GSM technology. In: Proceeding of the Second Joint EMBS/BMES Conference. Houston, October.

Gao, T., Pesto, C., Selavo, L., Chen, Y., Ko, J., Lim, J.H., ..., \& Welsh, M. (2008). Wireless medical sensor networks in emergency response: Implementation and pilot results. In: 2008 IEEE Conference on Technologies for Homeland Security (pp. 187-192). IEEE.
Hu, F., Lakdawala, S., Hao, Q., \& Qiu, M. (2009). Low-power, intelligent sensor hardware interface for medical data preprocessing. IEEE Transactions on Information Technology in Biomedicine, 13, 656-663.

Kappiarukudil, K.J., \& Ramesh, M.V. (2010). Real-Time Monitoring and Detection of "Heart Attack" Using Wireless Sensor Networks. In: 2010 fourth international conference on sensor technologies and applications (SENSORCOMM) (pp. 632-636). IEEE.

Kumekawa, J.K. (2000). Emerging trends in telehealth. Business briefing: Next generation healthcare, 62-64.

Lupu, R.G. (2003). Solution for home care monitoring via GSM network (master degree thesis). Naples: University of Naples "Federico II", Dept. of Electronic Engineering and Telecommunucations, Biomedical Engineering Unit.

O’Donoghue, J., \& Herbert, J. (2006). Profile based sensor data acquisition in a ubiquitous medical environment. In: Fourth Annual IEEE International Conference on Pervasive Computing and Communications Workshops, 2006. PerCom Workshops 2006 (pp. 5-pp). IEEE.

Ogawa, H., Maki, H., Tsukamoto, S., Yonezawa, Y., Amano, H., \& Caldwell, W. (2010). A new drip infusion solution monitoring system with a free-flow detection function. In: Annual International Conference of the IEEE Engineering in Medicine and Biology Society Conference proceedings (pp. 1214-1217).

Qureshi, A., Shoeb, A., \& Guttag, J. (2005). Building a high-quality mobile telemedicine system using network striping over dissimilar wireless wide area networks. In: 27th Annual International Conference of the Engineering in Medicine and Biology Society, 2005. IEEE-EMBS 2005 (pp. 3942-3945). IEEE.

Rajan, S.P., \& Rajamony, S. (2013b). Viable investigations and real-time recitation of enhanced ECG-based cardiac telemonitoring system for homecare applications: a systematic evaluation. Telemedicine and e-Health, 19, 278-286.

Rajan, S.P., \& Sukanesh, R. (2013a). Experimental Studies on Intelligent, Wearable and Automated Wireless Mobile Tele-Alert System for Continuous Cardiac Surveillance. Journal of Applied Research and Technology, 11, 133-143.

Rajan, S.P., Sukanesh, R., \& Vijayprasath, S. (2012). Analysis and effective implementation of mobile based tele-alert system for enhancing remote health-care scenario. HEALTHMED, 6, 2370-2377.

Rasid, M.F.A., \& Woodward, B. (2005). Bluetooth telemedicine processor for multichannel biomedical signal transmission via mobile cellular networks. IEEE Transactions on Information Technology in Biomedicine, 9, 35-43.

Suman, M.C., \& Prathyusha, K. (2012). Wireless ECG System Based on ARM LPC 2103 Processor. International Journal of Electronics and Communication Technology, 3, 115-119.

Tahat, A.A. (2008). Implementation of an sms-based telemedicine system for patient electrocardiogram monitoring. Proceedings of the 4th IASTED International Conference on Telehealth and Assistive Technologies, Telehealth/AT 2008 (pp. 223-228).

Toral, S.L., Quero, J.M., Pérez, M.E., \& Franquelo, L.G. (2001). A microprocessor based system for ECG telemedicine and telecare. ISCAS 2001-2001 IEEE International Symposium on Circuits and Systems Conference Proceedings (pp. 526-529).

Voskarides, S.C., Pattichis, C.S., Istepanian, R., Michaelides, C., \& Schizas, C.N. (2003). Practical evaluation of GPRS use in a telemedicine system in Cyprus. In: 4th International IEEE EMBS Special Topic Conference on Information Technology Applications in Biomedicine, 2003 (pp. 3942). IEEE.

Widya, I., Van Halteren, A., Jones, V., Bults, R., Konstantas, D., Vierhout, P., \& Peuscher, J. (2003). Telematic requirements for a mobile and wireless healthcare system derived from enterprise models. In: Proceedings of the 7th International Conference on Telecommunications, 2003. ConTEL 2003 (Vol. 2, pp. 527-534). IEEE.

Woodward, B., Istepanain, R.S.H., \& Richards, C.I. (2001). Design of the new telemedicine system using a mobile telephone. IEEE Trans. inf. Tech in Biomedicine, 5, 13-15.

Xiao, Y., Gagliano, D., LaMonte, M., Hu, P., Gaasch, W., Gunawadane, R., \& Mackenzie, C. (2000). Design and evaluation of a real-time mobile telemedicine system for ambulance transport. Journal of High Speed Networks, 9, 47-56. 